\title{
Note on the Radion Effective Potential in the Presence of Branes
}

\author{
Peng Wang ${ }^{1 *}$ and Xin-He Meng ${ }^{1,2} \dagger$ \\ 1. Department of Physics, Nankai University, Tianjin, 3000`1, P.R.China \\ 2. Department of Physics, University of Arizona, Tucson, AZ 85721
}

\begin{abstract}
In String Theory compactification, branes are often invoked to get the desired form of the radion effective potential. Current popular way of doing this assumes that the introduction of branes will not modify the background geometry in an important way. In this paper, we show by an explicit example that at least in the codimension 2 case, the gravitational backreaction of the brane cannot be neglected in deriving the radion effective potential. Actually, in this case, the presence of branes will have no effect on the dynamics of radion.
\end{abstract}

PACS: 11.25.Mj, 04.50.+h, 98.80.Cq, 98.80.Jk

\footnotetext{
* E-mail: pewang@eyou.com

† E-mail: xhm@ physics.arizona.edu
} 
Current quantum gravity theories, like String Theory, often invoke extra dimensions as a basic ingredient of the nature of spacetime. In order for those theories to be phenomenologically acceptable, one commonly assumes that the extra dimensions are compactified with a small and stabilized volume. So the study of compactification mechanisms and their stability are of central importance in those theories (see, e.g., Ref.[1] for a recent review).

In this paper we will focus on the dynamics of the volume of the extra dimensions. In many String Theory compactification scheme, this will be the only moduli that is left unfixed (see, e.g., Ref.[2]). For this we have to obtain the 4-dimensional effective potential for the radion, which is the 4-dimensional field that corresponds to dilatations of the compact dimensions. Recently, the discussion of radion effective potential received a lot of interest also because its central importance in exploring the String Theory "Landscape" [3] and in the attempt of constructing de Sitter vacuum in String Theory 2] in view of the recent cosmological observation that our Universe is now accelerating [4]. Radion effective potential can also help us construct models that may account for the current cosmic acceleration such as the construction in Ref. [5].

When discussing the radion stabilization problem, one possible way to contribute a term to the radion effective potential is adding branes (see, e.g., Refs.[1, 6]). After adding a $p$-brane, it is often assumed that the contribution to the radion effective potential in the Einstein frame is of the form

$$
V_{p}(\phi)=\tilde{\sigma} \exp \left(-\frac{2 n+3-p}{n} \sqrt{\frac{2 n}{n+2}} \frac{\phi}{M_{4}}\right),
$$

where $\tilde{\sigma}$ is the 'renormalized' brane tension that have absorbed the volume and other numerical factors, $n$ is the number of extra dimensions, $M_{4}$ is the 4-dimensional reduced Planck mass. The implicit assumption underlying such a form of contribution is that the introduction of branes will not modify the background geometry in an important way, i.e. the gravitational backreaction of the branes can be neglected 6] (A recent important progress in String Theory just adopted this contribution of branes to uplift the negative minimum of the radion effective potential to a positive metastable minimum 2]). In this paper, we will show that this assumption cannot always be justified. At least when the spacetime dimension is 6 , the gravitational backreaction of the 3-branes on the background geometry can never be neglected. Actually, the backreaction will just cancel the contribution (11) and thus branes will have no effect on the dynamics of radion. We will prove our assertion by a 
well studied 6-dimensional example 7] for which the exact solution in the presence of branes is known [9].

To begin with, following the common formalism when discussing radion stabilization 1, 6, 7], let's decompose the coordinates into the four macroscopic dimensions $x^{\mu}$ and the two extra dimensions $y^{a}$, and consider an ansatz for which the geometry factorizes into a 4-dimensional metric $g_{\mu \nu}$ depending only on $x$ and a 2-dimensional metric $\gamma_{a b}$ depending only on $y$,

$$
d s^{2}=G_{A B} d X^{A} d X^{B}=g_{\mu \nu}(x) d x^{\mu} d x^{\nu}+b^{2}(x) \gamma_{a b}(y) d y^{a} d y^{b}
$$

where $A, B=0, \ldots, 5, \mu, \nu=0, \ldots, 3$ and $a, b=4,5, b$ is the radion field.

The model we will discuss is a $\mathcal{S}^{2}$ compactification manifold stabilized by a bulk cosmological constant and magnetic flux. This model is a special example of the mechanism in Ref. 10] and has recently been discussed in detail in Ref. 7, 8]. The bulk lagrangian is

$$
S_{6}=\int d^{6} X \sqrt{|G|}\left(\frac{1}{2} M_{6}^{4} R-\lambda-\frac{1}{4} F_{A B} F^{A B}\right)
$$

where $M_{6}$ is the 6 -dimensional reduced Planck mass and $\lambda$ is the 6 -dimensional vacuum energy density. The 2 -form field strength takes the form $F_{a b}=\sqrt{|\gamma|} B_{0} \epsilon_{a b}$, where $B_{0}$ is a constant and $\epsilon$ is the standard antisymmetric tensor. Other components of $F_{A B}$ vanish identically.

Let's define the Einstein frame metric $\tilde{g}_{\mu \nu}$ by the conformal transformation

$$
\tilde{g}_{\mu \nu}=b^{2} g_{\mu \nu}
$$

and define a new field $\phi$ by

$$
b=\exp \left(\phi / 2 M_{4}\right)
$$

where $M_{4}^{2}=M_{6}^{4} \mathcal{V}$ is the 4-dimensional reduced Planck mass and $\mathcal{V} \equiv \int d^{2} y \sqrt{|\gamma|}$ is the volume of the extra dimensions. It has been shown in Ref.[7] that, after dimensional reduction, the radion dynamics will be described in the Einstein frame by a standard scalar field theory with the effective potential,

$$
\tilde{V}=\lambda \mathcal{V}\left(-2 e^{-2 \phi / M_{4}}+e^{-\phi / M_{4}}+e^{-3 \phi / M_{4}}\right)
$$

where we have set $\lambda=\frac{B_{0}^{2}}{2}$. 
Now let's consider adding branes to the above scenario. In the simplest case which is also the case considered in the current literature [1, 6, 9], the branes are described by NambuGoto action (see Ref. 11] for an elegant review),

$$
S_{N G}=-\sum_{i} \int d^{4} x \sqrt{|g|} \sigma_{i}
$$

where $i$ labels the branes, $\sigma_{i}$ and $X_{i}$ are the tension and position of the $i$ th brane, respectively. The energy-momentum tensor of branes follow by varying $G^{A B}$ in the action (7)

$$
T_{A B}^{b}=-\sum_{i} \frac{\sigma_{i}}{\sqrt{\gamma}}\left(\begin{array}{cc}
g_{\mu \nu} & 0 \\
0 & 0
\end{array}\right) \delta^{(2)}\left(y_{i}\right)
$$

In Ref. 9], Carroll and Guica considered two equal tension branes located at the two poles of the $\mathcal{S}^{2}$ manifold. They showed that the brane tension will induce a deficit angle in the extra dimension, thus $\gamma_{a b}$ will be of the form

$$
\gamma_{a b} d y^{a} d y^{b}=a_{0}^{2}\left(d \theta^{2}+\alpha^{2} \sin ^{2} \theta d \phi^{2}\right)
$$

with $a_{0}$, the radius of the extra dimension, and $\alpha$ given by

$$
a_{0}^{2}=\frac{M_{6}^{4}}{2 \lambda}, \quad \alpha=1-\frac{\sigma}{2 \pi M_{6}^{4}} .
$$

Then let's us consider the problem of radion stabilization in the above scenario. From the ansatz (2) we have

$$
\begin{gathered}
\sqrt{|G|}=\sqrt{|g|} \sqrt{|\gamma|} b^{2} \\
R[G]_{\mu \nu}=R[g]_{\mu \nu}-2 b^{-1} \nabla_{\mu} \nabla_{\nu} b, \\
R[G]_{a b}=R[\gamma]_{a b}-\gamma_{a b}\left[b \nabla^{2} b+(\nabla b)^{2}\right], \\
R[G]=R[g]+b^{-2} R[\gamma]-4 b^{-1} \nabla^{2} b-2 b^{-2}(\nabla b)^{2} .
\end{gathered}
$$

The bulk energy-momentum tensor contains contributions from the bulk cosmological constant and the gauge field,

$$
T_{A B}^{b u l k}=T_{A B}^{\lambda}+T_{A B}^{F}
$$


for which the explicit forms are

$$
\begin{aligned}
& T_{A B}^{\lambda}=-\lambda\left(\begin{array}{cc}
g_{\mu \nu} & 0 \\
0 & b^{2} \gamma_{a b}
\end{array}\right) \\
& T_{A B}^{F}=-\frac{b^{-4}}{2} B_{0}^{2}\left(\begin{array}{cc}
g_{\mu \nu} & 0 \\
0 & -b^{2} \gamma_{a b}
\end{array}\right) .
\end{aligned}
$$

Then, the longitudinal component of the Einstein equations is

$$
\begin{aligned}
R[g]_{\mu \nu}-2 b^{-1} \nabla_{\mu} \nabla_{\nu} b & -\frac{1}{2} g_{\mu \nu}\left(R[g]+b^{-2} R[\gamma]-4 b^{-1} \nabla^{2} b-2 b^{-2}(\nabla b)^{2}\right) \\
& =\frac{1}{M_{6}^{4}} g_{\mu \nu}\left(-\lambda-b^{-4} \frac{B_{0}^{2}}{2}-b^{-2} \frac{\sigma}{\sqrt{|\gamma|}} \sum_{i} \delta^{(2)}\left(y_{i}\right)\right)
\end{aligned}
$$

and the transverse component is

$$
R[g]=2 b^{-1} \nabla^{2} b+\frac{1}{M_{6}^{4}}\left(2 \lambda-b^{-4} B_{0}^{2}\right) .
$$

Contracting Eq.(17) with $g^{\mu \nu}$ and substituting Eq.(18) into it, we can find that

$$
b^{-2} R[\gamma]=2 b^{-1} \nabla^{2} b+2 b^{-2}(\nabla b)^{2}+\frac{1}{M_{6}^{4}}\left(\lambda+\frac{3}{2} b^{-4} B_{0}^{2}+b^{-2} \frac{2 \sigma}{\sqrt{|\gamma|}} \sum_{i} \delta^{(2)}\left(y_{i}\right)\right) .
$$

With the help of the Gauss-Bonnet theorem,

$$
\int d^{2} y \sqrt{|\gamma|} R[\gamma]=8 \pi
$$

and from the solution (9), $\mathcal{V}=4 \pi a_{0}^{2} \alpha$, we can integrate Eq.(19) over the transverse coordinate. It can be found that the dependence on $\sigma$ will just cancel out and we can get the equation of motion of radion,

$$
2 b^{-1} \nabla^{2} b+2 b^{-2}(\nabla b)^{2}+\frac{\lambda}{M_{6}^{4}}\left(1-4 b^{-2}+3 b^{-4}\right)=0
$$

Through the conformal transformation (41) and the field redefinition (15), it can be checked that in the Einstein frame, Eq.(21) just transforms to the radion equation of motion without the brane described by the effective potential (6),

$$
\tilde{\nabla}^{2} \phi+\frac{\lambda \mathcal{V}}{M_{4}}\left(e^{-\phi / M_{4}}-4 e^{-2 \phi / M_{4}}+3 e^{-3 \phi / M_{4}}\right)=0
$$

Since the radion $\phi$ satisfies the same equation of motion, the radion effective potential in the presence of branes is still given by (6) , i.e. the presence of branes will contribute nothing 
to the radion effective potential irrespective of the value of the brane tension $\sigma$. By the way, this discussion also shows explicitly that after adding branes, the model (31) is still stable.

It is important to ask whether the above result can be generalized to other codimension 2 brane configurations. We will argue that there is good evidence to believe that the answer is yes. The essential step in the above derivation is a relationship between the sum of the brane tensions and the local curvature of the compactification manifold following from the Gauss-Bonnet theorem. This relationship is also directly responsible for one remarkable feature of the solution (91): if the brane tension is a constant, the 4-dimensional geometry is

independent of it [9] (This feature has interesting implications in addressing the cosmological constant problem. However, as recently discussed in Ref. 12], if the brane tension changes during a phase transition, the 4-dimensional geometry will fail to remain flat. Thus the independence of the 4-dimensional geometry on the brane tension is not a cosmologically realistic feature). So we can see that those two features are actually intimately related: they are both the direct results of the Gauss-Bonnet theorem on 2-dimensional manifold with deficit angles. Furthermore, as shown in Ref.[13], applying the 2-dimensional Gauss-Bonnet theorem to more general brane configurations, one can show that the independence of the brane geometry on the brane tensions is a general feature of codimension 2 branes. So we think this is strong indication that the independence of the radion effective potential on the brane tension is actually a general feature of codimension 2 branes.

In sum, in this paper we showed that using the formula (11) to derive the radion effective potential cannot be always justified. The validity of the formula (11) deserves careful examination in every specific models.

\section{Acknowledgement}

We would like to thank Sergei D. Odintsov and Liu Zhao for helpful comment on the manuscript. P.W. would especially like to thank Jeremie Vinet for clarifying discussion on the radion effective potential. P.W. would also like to thank James M. Cline and David Tong for helpful correspondence on this paper. X.H.M. has benefitted a lot by helpful discussions with D.Lyth, L.Ryder, X.P.Wu, and X.M.Zhang. This work is supported partly by ICSC- 
World Laboratory Scholarship and Doctoral Foundation of National Education Ministry.

[1] E. Silverstein, hep-th/0405068.

[2] S. Kachru, R. Kallosh, A. Linde and S. P. Trivedi, Phys.Rev. D 68 (2003) 046005 hep-th/0301240.

[3] L. Susskind, hep-th/0302219.

[4] A. Riess et al. Astrophys. J. 116 (1998) 1009 astro-ph/9805201; S. Perlmutter el al. Astrophys. J. 517 (1999) 565 astro-ph/9812133.

[5] S. Nojiri and S. D. Odintsov, Phys. Lett. B 576 (2003) 5 hep-th/0307071.

[6] S. B. Giddings, Phys.Rev. D 68 (2003) 026006 hep-th/0303031; S. B. Giddings and R. C. Myers, hep-th/0404220 I. Navarro and J. Santiago, hep-th/0405173.

[7] S. M. Carroll, J. Geddes, M. B. Hoffman and R. M. Wald, Phys. Rev. D 66 (2002) 024036 hep-th/0110149.

[8] U. Guenther and A. Zhuk, Phys. Rev. D 56 (1997) 6391 gr-qc/9706050; ibid, Class. Quant. Grav. 15 (1998) 2025 gr-qc/9804018; ibid, Phys. Rev. D 61 (2000) 124001 hep-ph/0002009; ibid, Class. Quant. Grav. 181441 hep-ph/0006283; U. Guenther, A. Starobinsky and A. Zhuk, Phys. Rev. D 69 (2004) 044003 hep-ph/0306191; U. Guenther, P. Moniz and A. Zhuk, Phys. Rev. D 68 (2003) 044010 hep-th/0303023.

[9] S. M. Carroll and M. M. Guica, hep-th/0302067.

[10] E. Cremmer and J. Scherk, Nucl. Phys. B 108 (1976) 409; P. G. Freund and M. A. Rubin, Phys. Lett. B 97 (1980) 233.

[11] B. Carter, Int. J. Theor. Phys. 40 (2001) 2099 gr-qc/0012036.

[12] J. Garriga and M. Porrati, hep-th/0406158.

[13] R. Sundrum, Phys. Rev. D 59 (1999) 085010 hep-ph/9807348. 